\title{
Nature of Variability in Saccades
}

\author{
Jeroen B. J. Smeets ${ }^{1}$ and Ignace T. C. Hooge ${ }^{2}$ \\ ${ }^{1}$ Neurowetenschappen, Erasmus MC, NL-3000 DR Rotterdam; and ${ }^{2}$ Psychonomie, Universiteit Utrecht, NL-3584 CS Utrecht, \\ The Netherlands
}

Submitted 3 December 2002; accepted in final form 5 February 2003

Smeets, Jeroen B. J. and Ignace T. C. Hooge. Nature of variability in saccades. J Neurophysiol 90: 12-20, 2003. First published February 12,$2003 ; 10.1152 / j n .01075 .2002$. We studied the variability in saccades by comparing the peak velocities of saccades with the same target amplitude made with different actual amplitudes. We tested three hypotheses: the pulse-height noise hypothesis (peak velocity and amplitude vary proportionally), the localization noise hypothesis (variability in amplitude and peak velocity lie along the main sequence), and the independent noise hypothesis (variability in amplitude and peak velocity are independent). We measured eye orientation in two experiments by a scleral coil and a video system. Surprisingly, the main source of variability of saccades depended on the measurement system used. A combination of localization noise and independent noise best describes the data obtained by the video system. The independent noise (e.g., measurement inaccuracy) was the main source of variability. For the scleral coils, the variability was considerably larger than for the less accurate video system. The pulse-height noise hypothesis best describes this additional variability. Therefore we conclude that pulse-height noise is the main source of variability in saccades measured with scleral coils. We discuss the influence of scleral coils on saccade generation and suggest that a change in motor strategy due to the discomfort of wearing the coils might be the cause of the increased variability.

\section{N T R O D U C T I O N}

When saccades are made repeatedly to the same target, the saccade is never the same. Various characteristic parameters of the saccade as amplitude and peak velocity show variation between trials in an experiment. The question we address in this paper is where this variability originates. To answer this question, we distinguish various sources of variability in peak velocity and amplitude of saccades. As we will explain, these various sources of variability have different effects on the relatively fixed relationship between the amplitude of a saccade and its peak velocity (the main sequence) (Bahill et al. 1975). Measuring this relationship in a situation in which the variations in amplitude are mainly due to variations in the neural processing (i.e., in a full-cue condition using an accurate measurement system) can thus reveal what the main source of the neural variability is. We will study the variability in information processing for eye movements by comparing the peak velocities of saccades with different actual amplitudes made with the same target amplitude.

The variability we want to study is that in the execution of saccades. There is an infinite number of ways that the execu-

Address for reprint requests: J.B.J. Smeets, Afdeling Neurowetenschappen, Erasmus MC, Postbus 1738, NL-3000 DR Rotterdam, The Netherlands (E-mail: j.smeets@erasmusmc.nl). tion may be inaccurate. Moreover, the planning and the measurement also will introduce inaccuracies. Despite these difficulties, we hypothesized that the variability in saccade execution is large enough to be distinguished from the other two sources of variability (planning and measurement). This hypothesis is based on our interpretation of raw main sequence data published by others (Goossens and van Opstal 1997). We re-plotted their data and main sequence fit in Fig. $1 A$ and added dashed lines as our interpretation: the variations in peak velocity seem proportional to those in amplitude. We observed a similar pattern when plotting a main sequence from our earlier experiments (Tabak et al. 1996).

Here we will derive an extremely simple model of saccade execution that captures this pattern. We will refer to this model as the pulse-height noise hypothesis. For this model, we assume that such motor variability is caused by noise in the firing intensity of motoneurons (Harris and Wolpert 1998). In terms of a simple pulse-step model of saccade generation, the variability is only in the height of the pulse and not in its duration. We make the additional simplification that the duration of a saccade depends on the duration (and not the amplitude) of the motor command. If the noise in the final neural control signal results in a larger amplitude, the average velocity must vary proportionally. As the skewness of saccades varies only slowly with amplitude (van Opstal and van Gisbergen 1987), we can assume that the ratio between peak velocity and average velocity remains the same (our 2nd assumption), we predict that the peak velocity varies proportionally with amplitude (Fig. $1 B)$.

A second source of variability is inaccuracy of target determination by the experimental subject: the intended end-position of a saccade may vary from trial to trial. This might be caused by inaccurate target localization by the subjects but also by other causes. Examples are misperception of the initial orientation of the eye or properties of the collicular motor map (van Opstal and van Gisbergen 1989). If this kind of variability is the main cause of variability in saccade amplitude, the measured saccade amplitude will determine the peak velocity of a saccade, irrespective of the target amplitude. For instance, a $10^{\circ}$ saccade that undershoots an $11^{\circ}$ target should have the same peak velocity as a $10^{\circ}$ saccade that overshoots a $9^{\circ}$ target (the 2 sets of data points overlap in Fig. 1C). Formulated in other words, all saccades should fall on the main sequence. Consequently, there should be a clear correlation between the

\footnotetext{
The costs of publication of this article were defrayed in part by the payment of page charges. The article must therefore be hereby marked "advertisement" in accordance with 18 U.S.C. Section 1734 solely to indicate this fact.
} 

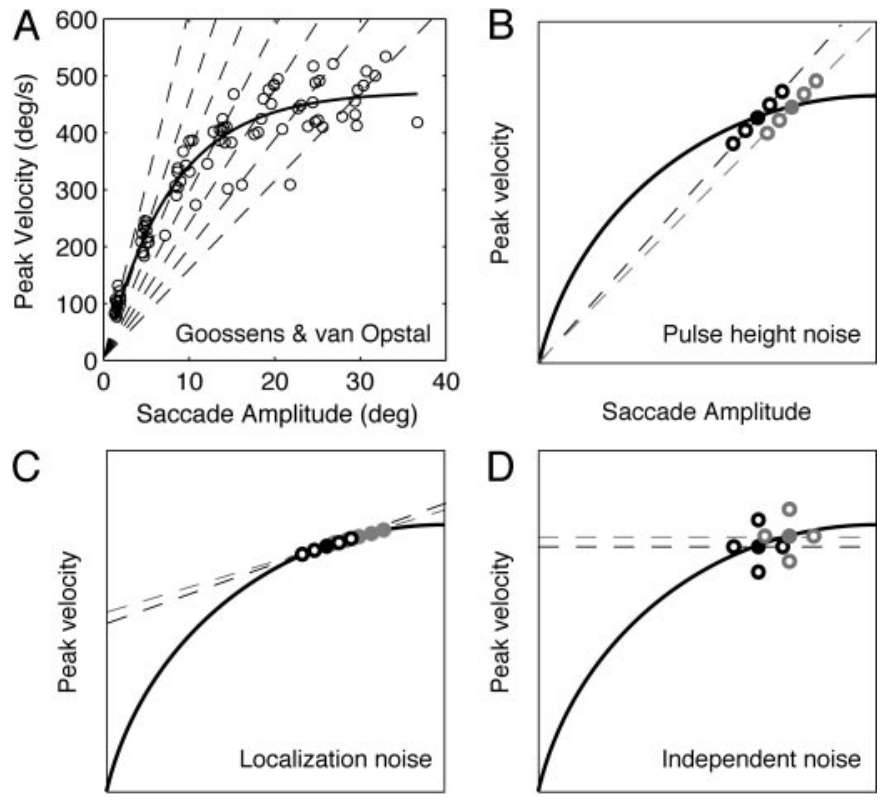

Saccade Amplitude

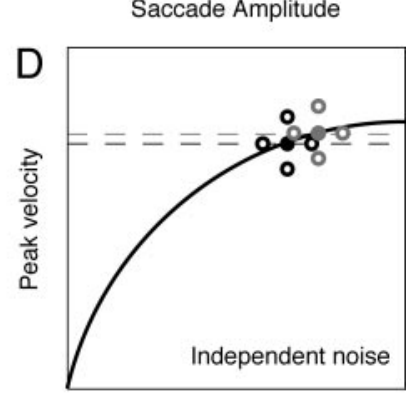

Saccade Amplitude

FIG. 1. Main sequence relationships. A: data for human eye saccades to visual targets at 7 eccentricities, with best fitting exponential (adapted with permission from Fig. 8 of Goossens and van Opstal 1997). - - -, our interpretation of the scatter of the data points around the fit. $B-D$ : scheme of the three predictions for the relationship between the variability in amplitude and peak velocity of saccades to targets at 2 eccentricities. $\bullet$, average for 1 target amplitude. - - , the correlation expected between variability in peak velocity and amplitude of saccades to a single target.

peak velocity and amplitude of saccades to a target at a certain distance, but the slope will be much shallower than for pulseheight noise. We will refer to this hypothesis as the localization noise hypothesis.

A last source of variability is measurement inaccuracy. For the determination of the main sequence, two parameters should be extracted from the eye-movement data: the saccade amplitude and the peak velocity of the saccade. We assume that measurement errors in the amplitude are determined by the resolution of the measurement of eye orientations. The bandwidth and the resolution of the measurement will determine the accuracy in determination of peak velocity. Because the peak velocity is determined at another instant as the orientations used for determining saccade amplitude (and assuming that these times are separated more than 1 over the bandwidth of the measurement system), it seems safe to assume that measurement noise causes thus independent variability in both measures (Fig. 1D). If independent variability in the determination of amplitude and peak velocity (measurement noise) is the main source of variability, the target amplitude will determine the peak velocity of a saccade, irrespective of the measured saccade amplitude. The subset of saccades that undershoot the target will have (on average) the same peak velocity as the subset of saccades that overshoots the same target. In fact, this holds for any other subset of saccades selected on the basis of their actual amplitude. Consequently, measurement noise will not lead to a correlation between peak velocity and saccade amplitude for saccades with the same target amplitude. We will refer to this hypothesis as the independent noise hypothesis.

In the discussion of the data analysis presented here, we have neglected two aspects. The first one is that variability in

one parameter can be compensated for (Quaia et al. 2000). In terms of a simple pulse-step model of saccade generation: variability in the initial pulse height might be compensated in a later part of the pulse. The result would be so that the peak velocity changes without a change in average velocity and thus without a change in amplitude. The consequence of this is that the variability in peak velocity will appear to be independent from the variability in amplitude. Compensated variability is thus indistinguishable from measurement noise.

The second neglected aspect is that more than one source of variability could play a role. For instance, adding ample independent (measurement) noise to data originating from a pulseheight noise system will result in regression with a slope equal to that of a regression to a pure localization noise system. We can distinguish between these two cases by taking into account the amount of variability. The localization noise hypothesis predicts that the coefficient of variation is larger for saccade amplitude than for peak velocity (the data points spread more in the amplitude than in the peak velocity in Fig. 1C). If we find a coefficient of variation that is larger for peak velocity than for saccade amplitude, we know that independent noise has to play an important role as it cannot be explained by one of the other hypothesis.

\section{METHODS}

This research is part of an ongoing research program that has been approved by the appropriate committees of both universities involved. During the course of the experiments, it became clear that scleral coils influence the dynamics of saccades (Frens and van der Geest 2002). We therefore performed the experiment not only with scleral coils (known to be the most accurate way) (Collewijn 1998) but also with a video-based system that has a much lower temporal and spatial resolution. As we will show, the two recording techniques not only differ in the accuracy but also influence the accuracy of the eye movements. To check whether these different results were indeed due to wearing the coils and not due to the differences in measurement accuracy, we performed an additional control experiment in which subjects were wearing coils while measuring the eye movements with the video-based system. In all experiments, we stabilized the head using a bite-board.

\section{Apparatus}

In the coil experiment, the orientation of the right eye was measured with an induction coil mounted in a scleral annulus (Skalar Medical, The Netherlands) in an AC magnetic field (Remmel Labs, Ashland, MA). This method was first described by Robinson (1963) and refined by Collewijn et al. (1975). The apparatus to generate the field and convert the coil-signal was developed by Remmel (1984). The horizontal and vertical eye orientations were measured at a sampling rate of $1,000 \mathrm{~Hz}$. The accuracy in determining saccade amplitude is about $0.05^{\circ}(\mathrm{V} 2$ times the SD in eye-orientation signal during fixation from our subject with the most stable fixation). The data were stored on the computer hard disk for off-line analysis.

In the video experiment, the orientation of the right eye was measured at $250 \mathrm{~Hz}$ with a video-based two-dimensional eye-tracking device (the Eyelink system, SR Research, Mississauga, Ontario, Canada). The accuracy in determining saccade amplitude for this system is about $0.08^{\circ}(\sqrt{ } 2$ times the $\mathrm{SD}$ in eye-orientation signal during fixation from our subject with the most stable fixation). Combined with the accuracy obtained for coils, we would expect that the inaccuracy in the angular difference between these two measurement systems would equal the root of the squared sum of the two inaccuracies, about $0.1^{\circ}$. By determining the SD in the amplitude difference 
over many saccades in many different directions, the relative accuracy of these systems has been estimated to be 10 times larger: about $1.0^{\circ}$ for $10^{\circ}$ saccades (Fig. 7 in van der Geest and Frens 2002). Part of the latter variability is due to systematic errors (such as a nonlinearity) that do not induce variability in our experiment (because we use only saccades between 2 positions). On the other hand, inaccuracy in determination onset and offset of saccades is not included in our first estimate of $0.08^{\circ}$. For peak velocity, van der Geest and Frens (Fig. 7) report an accuracy of the video system relative to scleral coils of $35 \%$ for $350 \%$ saccades. Sampled at $250 \mathrm{~Hz}, 35^{\circ}$ s corresponds to $0.14^{\circ}$ \% sample. As their velocity measure was based on a simple two-point difference between two samples, one can estimate the accuracy of the measurement of orientation differences with our video system to be about $0.14^{\circ}$. We will use this estimate in the remainder of this study. Note, however, that the accuracy of the system varies between subjects (van der Geest and Frens 2002).

In the two main experiments, targets were two 5-mm $\left(0.14^{\circ}\right)$ diameter dark blue disks fixed onto a light gray wall $2.0 \mathrm{~m}$ in front of the subjects. One target was fixed at about $5^{\circ}$ left of the subjective straight ahead. A second target could be positioned at $9-11^{\circ}$ (step: $0.5^{\circ}$ ) to the right of the first target. In the control experiment, the stimuli were presented on a monitor (resolution: $1,600 \times 1,200$ pixels; viewing distance, $80 \mathrm{~cm}$ ). For this experiment, we used the same target separations as in the coil and video experiment, but instead of keeping one target fixed at $5^{\circ}$, we varied the positions of both targets symmetrically around the subject's straight ahead.

The $10^{\circ}$ amplitude was chosen to have the clearest difference between the slopes predicted by the three hypothesis. The independent noise hypothesis predicts a zero slope, independent of saccade amplitude. The slopes predicted by the pulse-height noise and localization noise hypothesis both start at the same value, and both decrease to zero when the amplitude increases. However, the rate of decrease differs (this can be verified by differentiating the equation for the main sequence). The result is that difference between pulse-height noise and localization noise is the largest for saccades between 10 and $20^{\circ}$, and the difference between these two hypotheses and independent noise decreases with increasing amplitude. We therefore chose a separation between the targets of about $10^{\circ}$.

\section{Subjects}

The two authors participated in the two main experiments ( $A l$ and $A 2)$. Additionally, two colleagues (E1 and E2) of the Erasmus University participated the video experiment, and one colleague (U1) from the Utrecht University participated in the experiment using the scleral coil. These subjects were experienced subjects in eye-movement research but naive with respect to the questions of the present experiment. One author $(A I)$ and two other colleagues from the Utrecht University (U2 and $U 3$ ) performed the control experiment.

\section{Procedure}

Subjects were seated in a normally lighted laboratory. Before each session we calibrated the eye-movement apparatus by letting the subjects fixate an array of nine targets $\left(10.8 \times 8.0^{\circ}\right)$. Subsequently we presented the two targets and asked subjects to make saccades at a comfortable pace between them. To ensure that saccades started from a target, they were explicitly instructed to take their time to fixate the target before returning. Each 1-2 min, a new target separation was presented. The five target separations were presented pseudorandomly, and this sequence was presented twice. In this way, a possible systematic effect of fatigue on eye movements will not interfere with the results. We measured each subject's gaze for about $20 \mathrm{~min}$. Due to the time needed for calibration and manual set-up of the targets, this yielded 250-600 saccades per subject. In the control experiment, the automated stimulus presentation enabled us to measure 900-1300 saccades per subject.

\section{Data analysis}

Before saccade detection, the eye movement data were transformed from screen projections (video-data) and coil voltages (coil-data) to Fick angles. In Fick angles, the eye orientation is described by two angles. The first angle is a rotation about a vertical axis. The second rotation is a rotation about an eye-fixed horizontal axis (Haslwanter 1995).

As stated in the INTRODUCTION, the accuracy of the determination of the peak velocity is determined by limitations in sample frequency and measurement resolution. To be able to compare the velocities of the two set-ups with confidence, we determined for both measurement systems the eye velocity at 1-ms intervals based on position data from an 8 -ms time window. These velocities were obtained by fitting a parabola through $N$ subsequent data points ( $N$ is an odd number).

To obtain an 8-ms time window, $N$ was 3 for the data obtained in the video experiment. To be able to determine the velocity at each $\mathrm{ms}$, we used the derivatives of this fitted parabola at four instants (from 1 $\mathrm{ms}$ before the middle sample up to $2 \mathrm{~ms}$ after this sample) to estimate the value of the velocity at those instants. Coil data were measured at a sampling rate of $1,000 \mathrm{~Hz}$. To have the same $8-\mathrm{ms}$ time window as for the video data, $N$ was 9 for the measurements with the scleral coils. We subsequently determined the peak value in the $1,000 \mathrm{~Hz}$ velocity traces of each saccade. By using this method we were able to determine peak velocity on a millisecond time scale with the same time window for both systems. We expect that this results in a better relative accuracy than the $35 \%$ s reported by van der Geest and Frens (2002). As the velocities in the coil data are based on more samples of higher spatial accuracy, the accuracy will be higher than that of velocities in the video data. In the INTRODUCTION, we argued that we couldn't distinguish measurement noise from other sources of independent variability (e.g., compensated variability). For modeling purposes, we need to have an estimate of the total independent variability in peak velocity. We more or less arbitrarily assume independent uncertainty of peak velocity in the coil experiment of $20 \%$ s and in the video measurements of $25 \%$.

Saccade detection was done by a Matlab program that marked saccades by a velocity threshold of $75 \%$ s. After detection of all saccades, the program searched for onsets and offsets of the saccade. First it determined the averaged absolute velocity of a 100-ms period of fixation that started approximately $200 \mathrm{~ms}$ preceding the saccade. Onsets and offsets were determined by searching the time when absolute saccade velocity reached a value 3 SDs higher than the absolute velocity during the fixation.

If a saccade started or ended more than $5^{\circ}$ away from the targets or if the eye moved more than $1^{\circ}$ vertically during a saccade, this saccade was not further analyzed. Saccades were not further analyzed if the peak velocity did not occur between 25 and $75 \%$ of the movement. These criteria excluded 12 saccades from the video experiment (yielding 1,312 saccades) and 94 saccades from the coil-experiment (yielding 1,129 saccades).

Saccade amplitude was determined by taking the distance (in degrees) between the starting and landing positions of the saccade. This method is good enough to determine saccade amplitude because only small (of the order of $10^{\circ}$ ) horizontal saccades were analyzed. The last parameter we determined for each saccade was the target amplitude. This is the horizontal distance (in degrees) between the gaze at target onset and the center of the target.

We mentioned in the introduction that it is likely to have measurement noise in the determination of both the amplitude and the velocity. Interpreting the slopes from a regression analysis (as we will do) is therefore not without pitfalls. Variability in the independent variable will reduce the resulting slope of a linear least squares fit, whereas variability in the dependent variable does not affect the outcome (Fig. 2, $A$ and $B$ ). To get a regression that can be interpreted with confidence, one must classify the variable with the lowest signalto-noise ratio (the one with the largest variability) as dependent. To do 
A

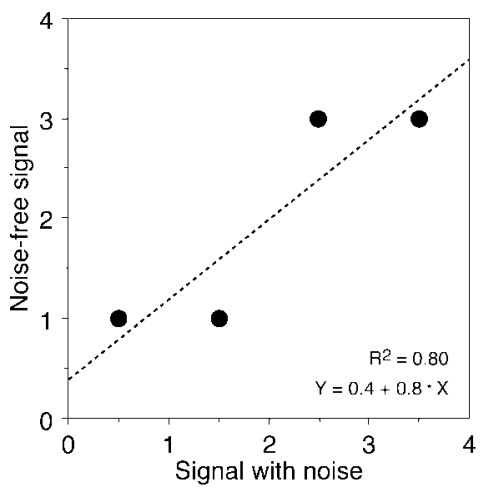

$\mathrm{C}$

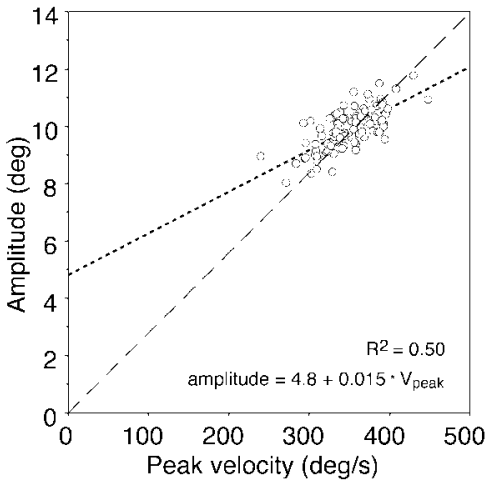

B

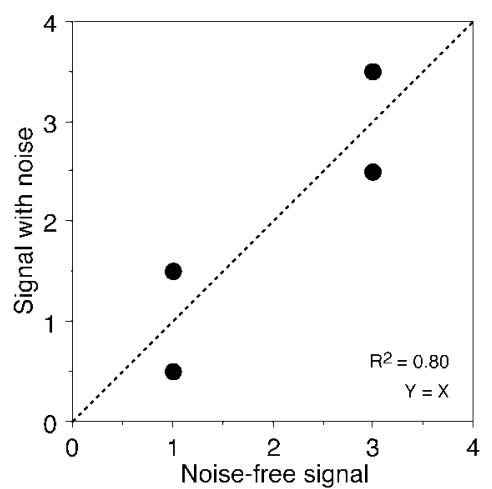

D

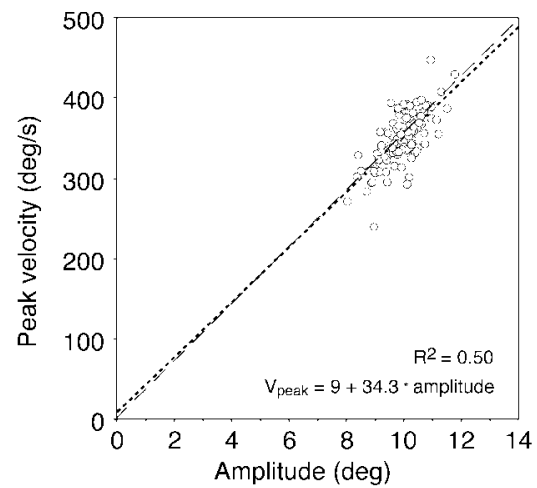

FIG. 2. The choice of the independent parameter influences the outcome of a regression analysis. $A$ and $B$ : regression to 4 data points with the variability either in the independent $(A)$ or dependent $(B)$ variable. The correlation coefficients are the same, but the slopes and intercepts differ considerably because a linear regression minimizes the vertical distance between the line and the data points. The regression theory assumes a situation as in $B$ in which all variability is in the dependent variable. Note that the sum of the squared residuals (vertical distances) is smaller with the erroneous choice of independent variable $(A)$. $C$ and $D$ : application to main sequence analysis. Two hundred model data points (the same set of points is used in both graphs) were generated according to the pulse-height noise model, with an average amplitude of $10^{\circ}$ and velocity $350 \%$. We assume that the pulse-height noise leads to a distribution of the saccades exactly along the dashed line with a SD of $6 \%$ in amplitude and velocity. We subsequently added normally distributed independent noise with SDs of $0.14^{\circ}$ to the amplitude and $25 \%$ s to the velocity values. We fitted a linear regression $(\cdots)$ line through the data using either variable as independent variable. The regression with peak velocity as independent variable $(C)$ suggests that pulse-height noise is not the main source of variability. However, if we use amplitude as the independent parameter $(B)$, the linear regression recovers the pulse-height noise model that underlies the data. so, we must estimate the amount of measurement noise (as opposed to behavioral variability). We will do so with a simple model, assuming that all measurement noise is due to limited accuracy in orientation measurement.

Although the reasoning in the following text holds irrespective of the particular accuracy value chosen, we make the reasoning simple by using the values we derived above for our video system. For $10^{\circ}$ saccades with a peak velocity of about $350^{\circ}$ s, we estimate that the accuracy of amplitude measurement is about $1.4 \%\left(0.14 \% 10^{\circ}\right)$, whereas it is about $7 \%$ for peak velocity. We illustrate the effect of choice of the independent variable with simulated data for the pulseheight model (assuming behavioral variability $1^{\circ}$ ) of Fig. $1 B$. The simulated measurements are plotted in Fig. 2, $C$ and $D$. It is clear that if we use amplitude as independent parameter, we can recover our behavioral model, but if we use velocity as independent parameter, the fit yields a different result as the model that generated the data. We therefore chose the amplitude as the independent parameter in our regression analysis.

The main data of the present study are the saccades with $9.5-10.5^{\circ}$ target amplitude. We will show two examples of velocity-amplitude relationships based on individual trials. Because our analysis yields more than thousand saccades for each experiment, we display the overall behavior after reducing the number of data points by averaging. To do so, we collected saccades into amplitude bins of $0.5^{\circ}$ wide and averaged the values for the peak velocity of all saccades in those bins [a method analogous to the one used for instance by Collewijn et al. (1988)]. Only the averages of bins containing more than three saccades will be displayed.

To check whether the variability of these saccades follows the main sequence, we had to determine the main sequence from our limited data set. To do so, we approximated this relationship by fitting a line through our data. Using a fit to the velocities and saccade amplitudes of all individual saccades would probably introduce a bias because the small saccades are in general undershooting the target, and large saccades overshooting (see our hypothetical data: Fig. $1 B, \bigcirc$ ). Thus the ends of the amplitude range would be dominated by aberrant saccades, which might have correspondingly aberrant kinematics, resulting in a distorted main sequence. We therefore chose to determine the main sequence for our saccades by a regression of peak velocity to target amplitude of all individual saccades (corresponding to Fig. 1, ). Subsequently, we compared this line with the tangent to the exponential main sequence fit shown in Fig. $1 A$ (Goossens and van Opstal 1997) at the applicable point $\left(10^{\circ}\right)$. This tangent is given by $V_{\text {peak }}=173+16.8 *$ amplitude.

\section{R E S U L T S}

As examples of individual behavior, we show the data of saccades with $9.5-10.5^{\circ}$ target amplitude for two of the naive subjects in Fig. 3. For subject $U 1$, measured with the coil system, the peak velocity seems proportional with the amplitude, as predicted by the pulse-height noise hypothesis (Fig. $1 B$ ). For subject $E 1$, measured with the video-based system, the relationship seems to be more close to the main sequence, the behavior predicted by the hypothesis of localization noise. Although the fit to the coil data explains much more of the observed variance than the fit to the video data $\left(r^{2}=0.79\right.$ vs. $r^{2}=0.23$ ), the residual SD in the peak velocity is almost the same in both examples (17.7 vs. $19.4 \%$ s).

To obtain an overall view of the behavior of all our subjects, we averaged the data over all subjects in $0.5^{\circ}$ wide bins (Fig. 4). The in this figure correspond to those in Fig. $1, B-D$, indicating the main sequence as a function of target amplitude. The $\bigcirc$ correspond to one set of $\bigcirc$ in Fig. $1, B-D$, indicating the variability in amplitude and peak velocity for one target amplitude $\left(9.5-10.5^{\circ}\right)$. The parameters of the various linear fits are given in Table 1. Both recording techniques enabled us to reproduce the main sequence relationship between (target) 


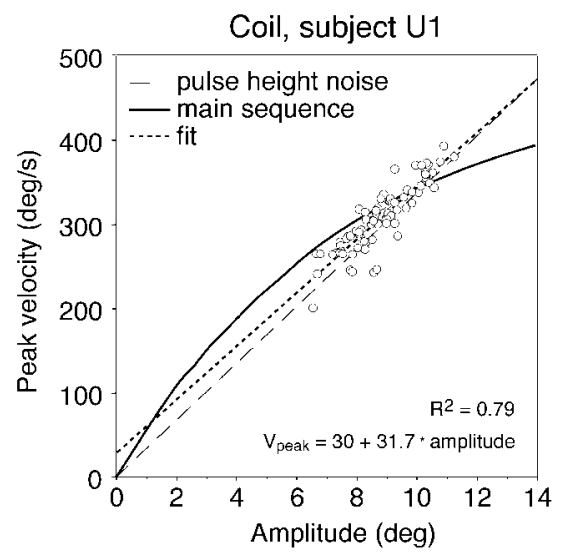

amplitude and peak velocity for all saccades reasonably well (O). We found some saccades with a target amplitude of less than $9^{\circ}$ and more than $11^{\circ}$, thus outside the range of target separations presented. Such target amplitudes can occur when the eye does not exactly fixate the fixation point before making a saccade.

However, when looking at the relationship between saccade amplitude and peak velocity for saccades with the same $10^{\circ}$ target amplitude $(\bigcirc)$, the two recording methods yielded different results. Using the scleral coils (400 saccades with 9.5$10.5^{\circ}$ target amplitude), the linear regression yielded an intercept close to zero (but significantly different, $P=0.001$ ). The slope of this linear fit is not significantly different from the value predicted by the pulse-height noise model. Using the video-based system (494 saccades with 9.5-10.5 ${ }^{\circ}$ target amplitude), however, the intercept differed clearly from zero, whereas the slope did not differ significantly from the slope of the tangent to the main sequence at $10^{\circ}$. We expect that the video system would lead to more measurement noise. Indeed, the variability in the peak velocity that is not explained by the linear fit (SD of the residuals of the fit, calculated over the data of all the subjects) is higher for the measurements with the video system than for those with the coils.

To test whether the measurement noise might have affected the slope of the regression lines, we looked at the coefficient of variation. If the different slopes in the two experiments are due to different sources of variability in behavior, we expect different ratio's between the coefficients of variation of peak velocity and that of amplitude. For localization noise (Fig. 1C), we expect a larger coefficient of variation for amplitude than for peak velocity. For pulse-height noise (Fig. 1B), we expect the same coefficient of variation for amplitude and peak velocity. We determined for each target amplitude and for each subject the coefficient of variation in both the amplitude and the peak velocity (Fig. $5 A$ ). For the scleral coils, the coefficient of variation does not differ between the two variables ( $t$-test, paired for each amplitude-bin and subject, $P=0.074$ ), in line with the predictions of pulse-height noise. For the video-based measurement, the coefficient of variation of amplitude is larger than that of peak velocity $(P=0.017)$, in line with the predictions of localization noise.

As opposed to what one would expect when measurement noise was the cause of variability, the variability in Fig. $5 A$ seems larger for the measurement with the more accurate scleral coils than for that with video. As this could be due to the different subjects used in the two experiments, we analyzed the values for the two subjects that participated in both experiments separately (in Fig. 5B). Paired $t$-test (pairing subjects and $0.5^{\circ}$ wide target-amplitude bins) showed that the differences in variability between the measurements of the two systems on these subjects were indeed significant, both for amplitude $(P=$ $0.04)$ and peak velocity $(P<0.0001)$. The measurements with scleral coils lead to a more variable behavior of these subjects than the measurement with the video system.

To rule out the possibility that the preceding results are due to some unknown artifact of comparing two measurement systems, we performed a control experiment. In this experiment, we measure the saccades with the video system and determined whether wearing scleral coils indeed influenced the variability in the way described in the preceding text. If so, the
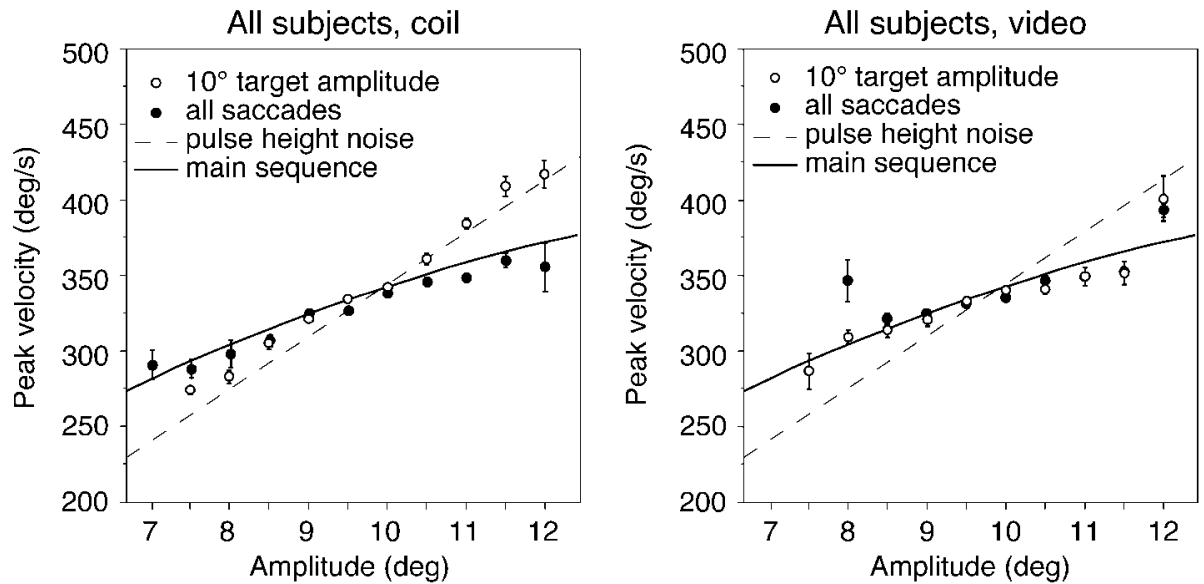

FIG. 4. Average behavior of our subjects measured with scleral coils (left) and video system (right). Peak velocities averaged for $0.5^{\circ}$ wide bins of amplitude as a function of amplitude. $\bullet$ the peak velocity of all saccades as a function of target amplitude. Both systems reproduce the main sequence for our range of amplitudes very well. $O$, the peak velocity of the saccades for which the target amplitude was between 9.5 and $10.5^{\circ}$ as a function of saccade amplitude. - and - - -, main sequence and pulse-height noise behavior predicted (see Table 1). 
TABLE 1. Summary of predictions, experimental results, and simulation results for the linear regressions of peak velocity as a function of saccade amplitude

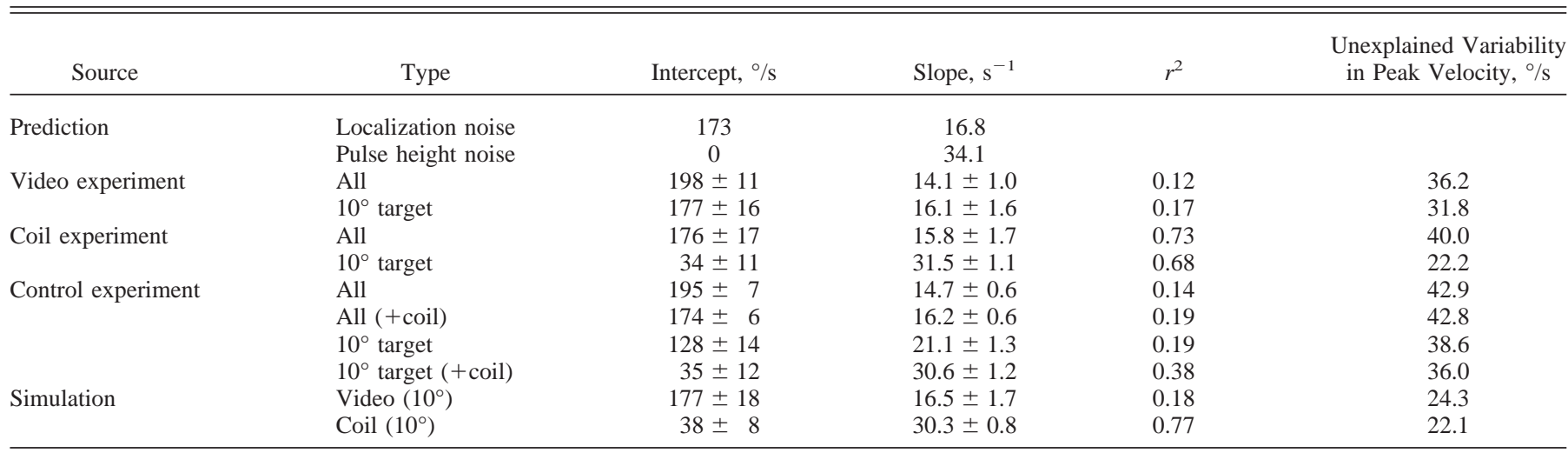

Values are means \pm SE. The prediction for the localization noise model is the tangent to the main sequence fit of Goossens and Van Opstal (1997) as shown in Fig. 1A. The prediction for the pulse height noise model is the line through the origin that yields the same velocity for $10^{\circ}$ saccades. The regression parameters in the last two rows are for a fit to 400 simulated saccades; see Fig. 8 for the parameters used for these two simulations.

video data of the subject wearing coils should resemble those of the ones measured with the coils, albeit with some additional variability.

As the assumed differential effect of two measurement systems on motor variability differed most clearly for $10^{\circ}$ target saccades, we have plotted the results of the original and control experiments in Fig. 6. The slope and the intercept of the velocity-amplitude relationships of saccades depends clearly more on whether there are coils in the eyes $(\boldsymbol{O}$ and $\bigcirc$ vs. $\boldsymbol{\square}$ and $\square)$ than on the measurement system used ( $\bigcirc$ vs. $९$ ). Table 1 shows that when comparing the two measurement systems for $10^{\circ}$ target saccades with coils in the eyes, the main difference between them is indeed the amount of unexplained variability in the saccade velocity.

\section{I S C U S S I O N}

The aim of this study was to find out what the main source of variability of saccades is. Our very simple pulse-height noise model can describe the variability of the most accurate system (the scleral coils) very well. The intercept of the regression to the $9.5-10.5^{\circ}$ target amplitude coil data (Table 1) differs slightly from zero, probably indicating a small effect of localization noise. Measurement noise is not an important factor in these measurements (the data lay close to the regression line in Fig. 3A) in accordance with the reported accuracy of this system. The variability in speed does not seem to be compensated to a large extent. If there was important compensation, this would result in lower variability in amplitude than in peak velocity. Our results show that the coefficients of variations do not differ between these variables when measured with coils (Fig. 5).

We thus confirmed our hypothesis based on our interpretation of raw main sequence plots (Goossens and van Opstal 1997). Pulse-height noise as the main source of variability can help to interpret another characteristic of velocity-amplitude relationships. It has been shown that by multiplying the peak velocity by the duration of the saccade, one obtains a variable that is proportional with amplitude with remarkably little scatter (van Opstal and van Gisbergen 1987). These authors conclude: "the considerable scatter in velocity-amplitude and amplitude-duration relation .. somehow cancels out" (p. 743). The explanation is simple: pulse-height noise. For pulse-height noise any deviation from the mean peak velocity is proportional to the deviation from the mean amplitude and will thus not be visible in a plot of peak velocity times duration against saccade amplitude. In Fig. 7, we illustrate this with a model example.

Surprisingly, we discovered that the answer discussed in the preceding text only holds when scleral coils are used (the experiments discussed in the previous paragraph were performed using scleral coils). For the less accurate measurement system, the outcome is different. The best fitting line is that of the localization noise hypothesis. Because the fit seems not that good, one might be tempted to assume that the shallow slope of the regression to the video data are caused by a combination of pulse-height noise and measurement noise. Two facts argue
A

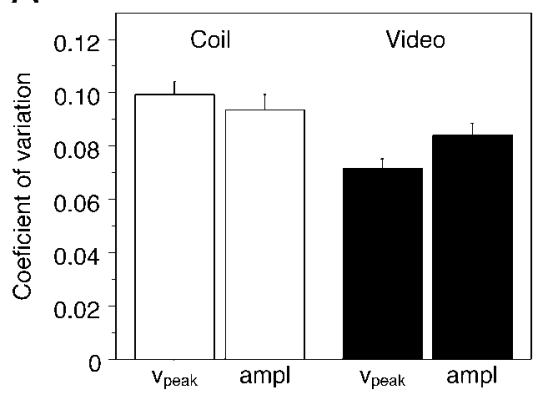

B

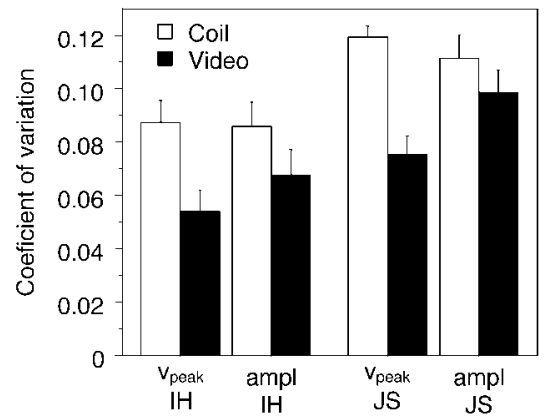

FIG. 5. The coefficient of variation for the amplitude and peak velocity of saccades. Error bars indicate SE across target amplitude and (in $A$ ) subjects. $A$ : the mean value for all the subjects. The variation in amplitude and in peak velocity is the same for the coil measurements, whereas the variation in amplitude is larger than that in the peak velocity for the video measurements. $B$ : for the 2 subjects that participated in both the coil experiment and the video experiment ( $A 1$ and $A 2$ ), the variability was larger when using scleral coils than when using the video system to measure eye movements. 


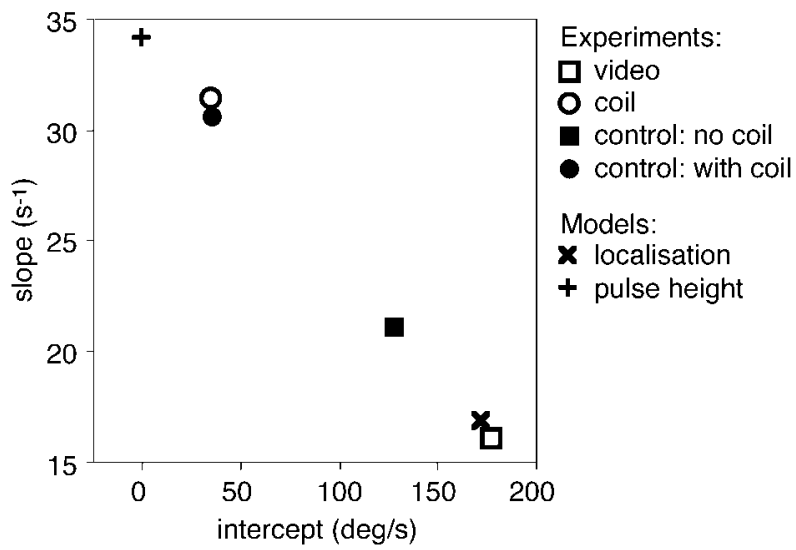

FIG. 6. The slopes and intercepts of velocity-amplitude plots for $9.5-10.5^{\circ}$ target amplitude saccades in the various experiments. When wearing coils ( and $\bigcirc$ ), the intercept and slope are close to the pulse-height noise model, independent of whether the saccades were recorded with the coils in the main experiment $(O)$ or with a video system in the control experiment $(\bullet) . x,+$, the predicted values for 2 of the models introduced in Fig. 1.

against this idea. First, the scatter around the regression line in the video data in Fig. $3 B\left(19.4^{\circ} / \mathrm{s}\right)$ is not much higher than that of the coil data in Fig. $3 A(17.7 \%$ s). The unexplained variability in peak velocity in a single subject is thus a bit lower than the independent variability in peak velocity we assumed $(7 \% \approx$ $25^{\circ} / \mathrm{s}$ ) in generating Fig. 2. Second, we showed (Fig. 2) that our analysis method could reveal pulse-height noise, even when over-estimating the independent variability (measurement inaccuracy) of the video-system.

A possible explanation that seems to be able to capture all aspects of our measurements is that the variability in the movements of the eye itself depends on the measurement method. More specifically: wearing scleral coils induces additional (pulse-height noise) variability. This explanation was confirmed by the control experiment. Furthermore, it is in line with results obtained with electrooculography: using that recording technique, the variations in peak velocity are independent from variations in saccade amplitude (Jürgens et al. 1981).

To check whether additional pulse-height noise indeed explains the differences between the coil experiment and the video experiment, we simulated 400 saccades using very simple models for the two experiments. We assume that all noise sources are normally distributed and characterize them by their $\mathrm{SD}$. For the both models, we assumed a localization noise $\left(0.7^{\circ}\right)$ (Kowler and Blaser 1995$)$, causing peak velocity variations along the main sequence [using the values of Goossens and van Opstal (1997)]. The independent noise is different for the two systems. Based on the characteristics of the apparatus used (see METHODS), we assumed for the video system an independent noise of $0.14^{\circ}$ for the amplitude and $25^{\circ}$ /s for peak velocity. We assumed that the coils were more accurate: $0.05^{\circ}$ for amplitude and $20 \%$ for peak velocity. For the coil model, we assume an additional $12 \%$ pulse-height noise. As can be seen in Table 1, the fit to the model saccades yields a very similar result as the fit to the experimentally measured saccades. In Fig. 8, we plotted a subset of 100 of the model saccades, comparable with the experimental results plotted in Fig. 3. Again, the correspondence between model predictions and experimental data are remarkable.

In the INTRODUCTION, we suggested three sources of variabil- ity in saccades. We can now summarize our results. For measurements with a video system, measurement noise contributes most to the variability in amplitude, and variability in execution (i.e., pulse-height noise) is negligible. For measurements with scleral coils, the measurement noise can be neglected. However, when wearing coils, the localization errors are not the main source of variability, as the coils induce additional neural variability, which follows the pulse-height noise model we hypothesized. This means that natural saccades are much less variable than one would conclude on the basis of measurements with scleral coils.

We can thus conclude that scleral coils induce extra variability in the eye movements. We are not the first ones to note the changes in eye movements induced by attaching coils. For instance, it has been shown that the implantation of coils reduces the gain of the VOR in mice (Stahl et al. 2000). They also reported an increase in variability (between animals) in the VOR-gain. What is the reason for the extra variability when using a scleral coil? If the coils would alter proprioceptive signals about eye orientation (Gauthier et al. 1990), one would expect additional localization noise and not additional pulseheight noise. There are two possible mechanisms that might be the cause of the extra variability. The first one is that the scleral coil changes the dynamical properties of the eye: it adds some inertia and might increase the viscous drag of the eye moving under the eyelid. The neural controller has to find a way to deal with this new situation, and the search for a new optimal control signal is reflected by variability in performance. However, the additional inertia of the coil is so small $(85 \mathrm{mg})$ that
A
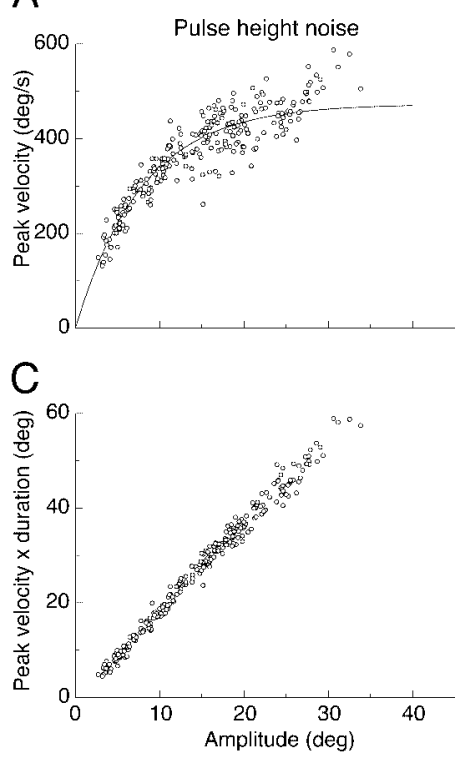

B

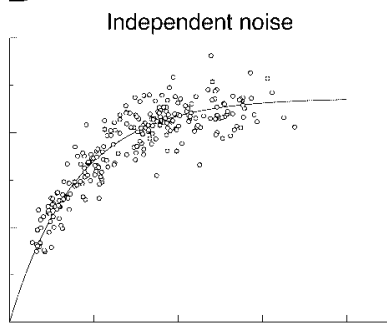

D

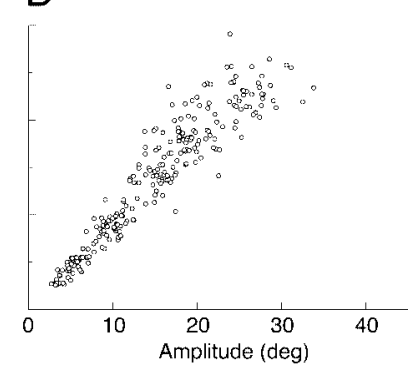

FIG. 7. Model simulation of 250 saccades with target amplitudes ranging from 5 to $30^{\circ}$. The mean values of peak velocity and duration were a function of amplitude following the fit results of Goossens and Van Opstal (1997). For $A$ and $C$ (pulse-height noise) we used the same sources of variability for peak velocity and amplitude as for the calculations for the coils in Fig. 8. For $B$ and $D$, we replaced the $12 \%$ pulse-height noise by $3.5 \%$ independent noise in peak velocity and amplitude. The duration of saccades in the model was not affected by noise. Although the scatter around the main sequence is the same for both model systems (top), the scatter in the relation between peak-velocity times duration and amplitude is clearly different. The scatter in $C$ is very similar to the scatter in the graphs in Fig. 8 of Van Opstal and Van Gisbergen (1987). 

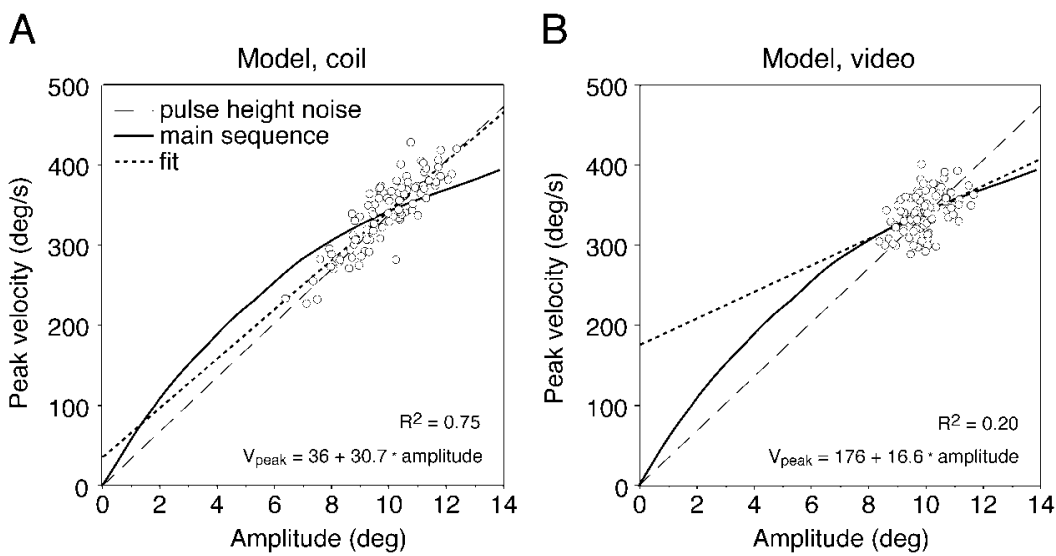

FIG. 8. Velocity-amplitude plots for 100 simulated responses to $10^{\circ}$ target amplitudes. For the model, we assumed a localization noise of $0.7^{\circ}$ for both measurement systems. The independent noise in amplitude is $0.05^{\circ}$ for the coil and $0.14^{\circ}$ for the video. The independent noise in peak velocity is $20 \%$ for the coil and $25 \%$ for the video. For the coil model, we assume an additional $12 \%$ pulse-height noise. Note the similarity of these simulated velocity-amplitude relationships to the actual data plotted in Fig. 3. the changed inertia does not seem a very likely explanation (Frens and van der Geest 2002).

Another explanation is that wearing a scleral coil changes the motor strategy of saccades in such a way that not only systematic changes in saccade parameters occur (Frens and van der Geest 2002) but also in some way extra variability in the subjects' behavior. This might seem unlikely but is indirectly supported by some recent papers. It has been shown that changing the motor strategy of a subject changes the characteristics of saccades systematically. Steinman et al. (1990) showed that applying a bite-board reduces the peak velocity of eye movements to a much larger extent than could be explained by the contribution of the head movement. Epelboim et al. (1997) showed that when subjects moved their hand to the target of a saccade, the peak velocity of the saccades was increased systematically. No mention of variability was made in these studies. In monkeys, Takikawa et al. (2002) used another manipulation that increased the nuisance of the monkey: removing the reward. Again, a reduction of peak velocity was observed. But more importantly: the variability in both the amplitude and velocity was increased due to the removal of rewards. These studies suggest that the variability of saccades depends on the motor strategy chosen and that a strategy that results in lower peak velocities and more variability is chosen in more restrained and uncomfortable situations.

The preceding reasoning might suggest that attaching a coil to the sclera of a human eye would have a different effect on saccades than implanting coils in the eyes of a monkey. However, several studies have shown that the slope of saccades made to a single target differs clearly from the local tangent to the main sequence. For $20^{\circ}$ saccades, a slope of about $30 \mathrm{~s}^{-1}$ has been reported (Snyder et al. 2002), almost twice the tangent to the main sequence in monkeys (assuming an exponential described by $1,000^{\circ} / \mathrm{s}$ saturation and $12.4^{\circ}$ for the exponential coefficient). Inspection of other speed amplitude plots of monkey saccades systematically shows a steeper slope for saccades to a single target than for saccades over a larger range (Fig. $4 \mathrm{~A}$ of Frens and van Opstal 1997; Fig. 3 of Quaia et al. 2000; Fig. $8 A$ of Straube et al. 1997; Fig. $6 A$ of White et al. 1994). These results indicate that uncompensated pulse-height noise plays also an important role in the variability in saccades made by non-human primates with implanted coils.

In the present paper, we investigate the causes of the variability in saccades. This question is closely related to that of (Quaia et al. 2000), who investigated whether variability in peak velocity was compensated. Based on their experiments with monkeys, they conclude that the saccadic system does not completely compensate for the variability in velocity, but only for about $60 \%$. How does their result correspond with ours? Our independent noise hypothesis corresponds to $100 \%$ compensation in their terminology; pulse-height noise corresponds to no compensation, and localization noise corresponds to a negative compensation. In their terminology, we find thus zero percent or less compensation when measuring with coils, and Snyder et al. (2002) found negative compensation in their monkeys. Both are even stronger conclusions than Quaia et al. (2000) made.

We think that this difference in conclusion is at least partly caused by two shortcomings with the methods of Quaia et al. (2000). First they used all saccades between a fixation point and a target as a group with the same target amplitude. As the eye does not exactly fixate the fixation point before making a saccade, not only the saccade amplitude but also the target amplitude will vary between trials. That is why one sees for our data in Fig. 4 some saccades with a target amplitude () of $<9^{\circ}$ and more than $11^{\circ}$, thus outside the range of target separations presented. A second problem with their analysis is that they used peak velocity as independent parameter. Figure 2 shows how that can lead to a change in slope toward a line that would be interpreted as perfect compensation. If we analyze our model data of Fig. $2\left(10^{\circ}\right.$ saccades without compensation) in the way of Quaia et al. (2000), we find $47.5 \%$ compensation. This is the value they found for saccades of the same amplitude $\left(10^{\circ}\right)$ in two of their three monkeys. So, the experimental results of Quaia et al. (2000) might not even differ from ours. Therefore the results of their analysis do not exclude a complete absence of compensation for variability in velocity.

Our conclusion that wearing scleral coils induces extra variability in eye movements is a rather disappointing message for the eye-movement research community. Scleral coils have always been regarded as the most precise measurement system. However, a measurement system that decreases human precision is not as accurate as its technical precision. This means that scleral coils cannot be regarded as the golden standard for accuracy in eye movement research anymore. However, the alternative systems are not ideal either. The video-based systems have (at present) two disadvantages. The first one is that they do not measure orientation directly, but the effects of changing orientation on the projection of the eye's image. This makes coils at present the ideal tool for measuring threedimensional eye movements (Hooge and van den Berg 2000). 
A second disadvantage of a video-based system is that they do not measure the orientation of the eye in space but relative to the head. Correction of the data for head movements is far from trivial. Moreover, measurements with a moving head are fundamentally perturbed as the connection between camera and head is not completely rigid. As coils measure the orientation of the eye in space, they are still the only tool to accurately measure the function of the vestibuloocular reflex (Collewijn and Smeets 2000). It has been recently shown that for most other tasks, video-based systems are good enough (van der Geest and Frens 2002), and even preferable because coils slow down saccades (Frens and van der Geest 2002). We now show that there is another reason to prefer video-based system to coils because the latter increase the variability in performance.

We like to thank J. van der Geest for the Matlab routine to determine instantaneous velocity and R. van Wezel and C. Erkelens for the use of the coil set-ups.

\section{REFERENCES}

Bahill AT, Clark MR, and Stark L. The main sequence, a tool for studying human eye movements. Math Biosci 24: 191-204, 1975.

Collewijn H. Eye movement recording. In: Vision Research, A Practical Guide to Laboratory Methods, edited by Carpenter RHS and Robson JG. Oxford, UK: Oxford Univ. Press, 1998, p. 245-285.

Collewijn H, Erkelens CJ, and Steinman RM. Binocular co-ordination of human horizontal saccadic eye movements. J Physiol 404: 157-182, 1988.

Collewijn $\mathbf{H}$ and Smeets JBJ. Early components of the human vestibuloocular response to head rotation: latency and gain. J Neurophysiol 84: 376-389, 2000.

Collewijn H, van der Mark F, and Jansen TC. Precise recording of human eye movements. Vision Res 15: 447-450, 1975.

Epelboim J, Steinman RM, Kowler E, Pizlo Z, Erkelens CJ, and Collewijn H. Gaze-shift dynamics in two kinds of sequential looking tasks. Vision Res 37: 2597-2607, 1997.

Frens MA and van der Geest JN. Scleral search coils influence saccade dynamics. J Neurophysiol 88: 692-698, 2002.

Frens MA and van Opstal AJ. Monkey superior colliculus activity during short-term saccadic adaptation. Brain Res Bull 43: 473-483, 1997.

Gauthier GM, Nommay D, and Vercher JL. Ocular muscle proprioception and visual localization of targets in man. Brain 113: 1857-1871, 1990.

Goossens HHLM and van Opstal AJ. Human eye-head coordination in two dimensions under different sensorimotor conditions. Exp Brain Res 114: 542-560, 1997.
Harris CM and Wolpert DM. Signal-dependent noise determines motor planning. Nature 394: 780-784, 1998.

Haslwanter T. Mathematics of three-dimensional eye rotations. Vision Res 35: 1727-1739, 1995.

Hooge ITC and van den Berg AV. Visually evoked cyclovergence and extended Listing's law. J Neurophysiol 83: 2757-2775, 2000.

Jürgens R, Becker W, and Kornhuber HH. Natural and drug-induced variations of velocity and duration of human saccadic eye movements: evidence for a control of the neural pulse generator by local feedback. Biol Cybern 39: 87-96, 1981.

Kowler E and Blaser E. The accuracy and precision of saccades to small and large targets. Vision Res 35: 1741-1754, 1995.

Quaia C, Paré M, Wurtz RH, and Optican LM. Extent of compensation for variations in monkey saccadic eye movements. Exp Brain Res 132: 39-51, 2000.

Remmel RS. An inexpensive eye movement monitor using the scleral coil technique. IEEE Trans Biomed Eng 31: 388-390, 1984.

Robinson DA. A method of measuring eye movement using a scleral search coil in a magnetic field. IEEE Trans Biomed Eng 10: 137-145, 1963.

Snyder LH, Calton JL, Dickinson AR, and Lawrence BM. Eye-hand coordination: saccades are faster when accompanied by a coordinated arm movement. J Neurophysiol 87: 2279-2286, 2002.

Stahl JS, van Alphen AM, and de Zeeuw CI. A comparison of video and magnetic search coil recordings of mouse eye movements. J Neurosci Methods 99: 101-110, 2000.

Steinman RM, Kowler E, and Collewijn H. New directions for occulomotor research. Vision Res 30: 1845-1864, 1990.

Straube A, Fuchs AF, Usher S, and Robinson FR. Characteristics of saccadic gain adaptation in Rhesus macaques. J Neurophysiol 77: 874-895, 1997.

Tabak S, Smeets JBJ, and Collewijn H. Modulation of the human vestibuloocular reflex during saccades: probing by high-frequency oscillation and torque pulses of the head. J Neurophysiol 76: 3249-3263, 1996.

Takikawa Y, Kawagoe R, Itoh H, Nakahara H, and Hikosaka O. Modulation of saccadic eye movements by predicted reward outcome. Exp Brain Res 142: 284-291, 2002.

van der Geest JN and Frens MA. Recording eye movements with videooculography and scleral search coils: a direct comparison of two methods. J Neurosci Methods 114: 185-195, 2002.

van Opstal AJ and van Gisbergen JAM. Scatter in the metrics of saccades and properties of the collicular motor map. Vision Res 29: 1183-1196, 1989.

van Opstal AJ and van Gisbergen JAM. Skewness of saccadic velocity profiles: a unifying parameter for normal and slow saccades. Vision Res 27: 731-745, 1987.

White JM, Sparks DL, and Stanford TR. Saccades to remembered target locations - an analysis of systematic and variable errors. Vision Res 34: 79-92, 1994. 